Research Article

\title{
Sinc Collocation Method for Solving the Benjamin-Ono Equation
}

\author{
Edson Pindza and Eben Maré \\ Department of Mathematics and Applied Mathematics, University of Pretoria, Pretoria 002, South Africa \\ Correspondence should be addressed to Eben Maré; eben.mare@up.ac.za
}

Received 22 March 2014; Accepted 17 June 2014; Published 23 July 2014

Academic Editor: Ali Cemal Benim

Copyright ( 2014 E. Pindza and E. Maré. This is an open access article distributed under the Creative Commons Attribution License, which permits unrestricted use, distribution, and reproduction in any medium, provided the original work is properly cited.

We propose a simple, though powerful, technique for numerical solutions of the Benjamin-Ono equation. This approach is based on a global collocation method using Sinc basis functions. Some properties of the Sinc collocation method required for our subsequent development are given and utilized to reduce the computation of the Benjamin-Ono equation to a system of ordinary differential equations. The propagation of one soliton and the interaction of two solitons are used to validate our numerical method. The method is easy to implement and yields accurate results.

\section{Introduction}

It is well known that nonlinear partial differential equations (NPDEs) are widely used to describe complex phenomena in various fields of sciences, such as physics, biology, and chemistry.

In this paper, we consider the initial value problem for the Benjamin-Ono (BO) equation of the form

$$
u_{t}+u u_{x}+\mathscr{H}\left(u_{x x}\right)=0, \quad(x, t) \in \mathbb{R}^{2}
$$

together with the following initial and the boundary conditions:

$$
u(x, 0)=u_{0}(x), \quad u, u_{x} \longrightarrow 0 \quad \text { as }|x| \longrightarrow \infty .
$$

Here $u: \mathbb{R}^{2} \rightarrow \mathbb{R}$ is a real valued function and $\mathscr{H}$ denotes the Hilbert transform defined and described by [1] (amongst others):

$$
\mathscr{H}[f](x)=\frac{1}{\pi} P \int_{-\infty}^{\infty} \frac{f(x-y)}{y} d y,
$$

where $P$ denotes the Cauchy principal value. This equation was derived by Benjamin [2] and later by Ono [3] as a model for one-dimensional waves in deep water and it has a close relation with the famous KdV equation which models long waves in shallow water [4].

We recall that the $\mathrm{BO}$ equation has an infinite sequence of invariants [5], the first three of which are

$$
\begin{aligned}
& I_{1}=\int_{-\infty}^{\infty} u d x \quad \text { (mass) } \\
& I_{2}=\int_{-\infty}^{\infty} u^{2} d x \quad \text { (momentum), } \\
& I_{3}=\int_{-\infty}^{\infty}\left[u^{3}-3 u_{x} \mathscr{H}(u)\right] d x \quad \text { (energy). }
\end{aligned}
$$

The BO has been shown to admit rational analytical soliton solutions in $x$ and $t$ [6]. However, for a given arbitrary initial condition, finding analytical solutions of the $\mathrm{BO}$ equation becomes an intractable problem. Therefore the use of numerical methods plays an important role in the study of the dynamics of the $\mathrm{BO}$ equation.

James and Weideman [7] used the Fourier method which implicitly assumes the periodicity of the boundary conditions and a method based on rational approximating function to compute numerical solutions of the $\mathrm{BO}$. The rational method was shown to have spectacular accuracy for solutions that do not wander too far from the origin while, for long dated 
solutions, the Fourier method was shown to retain superior accuracy. Miloh et al. [8] proposed an efficient pseudospectral method for the numerical solution of the weakly nonlinear Benjamin-Ono equation for arbitrary initial conditions and suggested a practical new relationship for estimating the number of solitons in terms of arbitrary initial conditions. Thomée and Vsaudeva Murthy [9] used the Crank-Nicolson approximation in time and finite difference approximations in space to solve the $\mathrm{BO}$ equation. They treated the nonlinear term in a standard conservative fashion and discretized the Hilbert transform by a quadrature formula which was computed efficiently using the fast Fourier transform. Recently, Boyd and $\mathrm{Xu}$ [10] compared three spectral methods for solving the Benjamin-Ono equation, Fourier pseudospectral, rational Christov functions, and Gaussian radial basis functions. They highlighted the advantages and the disadvantages of each numerical method. For instance, the Fourier pseudospectral method is very fast through use of the fast Fourier transform (FFT), but requires domain truncation which is unnecessary for a rational basis. Radial basis functions are slow for a given number of grid points; however, they have a very flexible grid adaptation. To the best of our knowledge, there are no records regarding numerical solutions of the BO equation by using Sinc numerical methods.

In this paper, we propose a Sinc collocation method for solving the BO equation. Sinc numerical methods for solving ordinary and partial differential equations have increasingly become popular and have been extensively studied, in particular, for problems on unbounded domains and those having singularity at boundaries. The first Sinc method was introduced by Stenger [11] to solve two-point boundary value problems for second order differential equations. In the field of nonlinear partial differential equations, the use of Sinc numerical methods has shown tremendous potential. For instance, Al-Khaled [12] showed the utility of the Sinc Galerkin method for solving the Korteweg-de Vries equation. Recently, Mokhtari and Mohammadi [13] presented a meshfree method based on the Sinc collocation method to solve the generalized regularized long wave equation. Excellent overviews of the existing Sinc methods for solving ODEs, PDEs, and integral equations can be found in $[14,15]$.

The layout of this paper is as follows. We describe the formulation of the Sinc collocation method and the discretization of the Hilbert transform in Section 2. Section 3 is devoted to the Sinc collocation discretization of the $\mathrm{BO}$ equation. Furthermore, we examine the stability of the method by using a linearized stability analysis. Numerical results illustrating the merits of the new scheme are given in Section 4. Finally, we present our conclusions in Section 5.

\section{Sinc Collocation Methods}

In this section, we introduce some useful definitions and pertinent theorems of the Sinc function needed for space discretization of the $\mathrm{BO}$ equation. We use $\mathbb{Z}$ to denote the set of all integers, $\mathbb{R}$ the set of all real numbers, and $\mathbb{C}$ the set of all complex numbers. Let $u$ be a function defined on $\mathbb{R}$ and $h$ the step-size. Then the Whittaker function is defined by the following series:

$$
C(u, h)(x)=\sum_{j=-\infty}^{\infty} u_{j} S_{j}(x)
$$

where $S_{j}$ is given as follows:

$$
S_{j}(x)=\operatorname{Sinc}\left(\frac{x-j h}{h}\right), \quad j=0, \pm 1, \pm 2, \ldots,
$$

and the Sinc function defined on the real line $\mathbb{R}$ is given by

$$
\operatorname{Sinc}(x)= \begin{cases}\frac{\sin (\pi x)}{\pi x}, & x \neq 0 \\ 1, & x=0\end{cases}
$$

Whenever the series given in (5) converges, $u$ is approximated by using a finite number of terms. Therefore, for a positive integer $N$, (5) implies

$$
C_{N}(u, h)(x)=\sum_{j=-N}^{N} u_{j} S_{j}(x) .
$$

The approximation of the $n$th derivative of the function $u$ by the Sinc is given by

$$
u^{(n)}(x) \approx \sum_{j=-N}^{N} u(j k) \frac{d^{n}}{d x^{n}}\left[S_{j}(x)\right]
$$

The Sinc collocation method requires derivatives of Sinc functions to be evaluated at collocation points. We can obtain explicit close form expressions for these; namely,

$$
\begin{gathered}
\delta_{j k}^{(0)}=\left.\left[S_{j}(x)\right]\right|_{x=x_{k}}= \begin{cases}1, & j=k \\
0, & j \neq k .\end{cases} \\
\delta_{j k}^{(1)}=\left.\frac{d}{d x}\left[S_{j}(x)\right]\right|_{x=x_{k}}= \begin{cases}0, & j=k, \\
\frac{(-1)^{j-k}}{h(j-k)}, & j \neq k .\end{cases} \\
\delta_{j k}^{(2)}=\left.\frac{d^{2}}{d x^{2}}\left[S_{j}(x)\right]\right|_{x=x_{k}}= \begin{cases}\frac{-\pi^{2}}{3}, & j=k, \\
\frac{-2(-1)^{j-k}}{h^{2}(j-k)^{2}}, & j \neq k .\end{cases}
\end{gathered}
$$

Lemma 1. The Hilbert transform of a function $u$ can be approximated by

$$
\mathscr{H}[u](x)=\frac{1}{\pi} P \int_{-\infty}^{\infty} \frac{f(x-y)}{y} d y \approx \sum_{j=-N}^{N} u_{j} T_{j}(x),
$$

where

$$
T_{j}(x)=\frac{1-\cos [\pi / h(x-j h)]}{\pi / h(x-j h)} .
$$


The entries of the Hilbert transform matrix at collocation point $x_{k}=k h$ are given by

$$
H_{j k}=T_{j}\left(x_{k}\right)= \begin{cases}0, & j=k, \\ \frac{1-(-1)^{j-k}}{\pi(j-k)}, & j \neq k .\end{cases}
$$

In order to state the convergence theorem of the Sinc collocation method, we introduce the following notation and definitions.

Definition 2. Let $\mathscr{D}_{d}$ denote the infinite strip region with width $2 d(d>0)$ in the complex plane

$$
\mathscr{D}_{d} \equiv\{z \in C|| \operatorname{Imz} \mid<d\} .
$$

For all $0<\varepsilon<1$, let $\mathscr{D}_{d}(\varepsilon)$ be defined by

$$
\mathscr{D}_{d}(\varepsilon)=\left\{z \in C|\operatorname{Rez}|<\frac{1}{\varepsilon},|\operatorname{Imz}|<d(1-\varepsilon)\right\} .
$$

Let $H^{1}\left(\mathscr{D}_{d}\right)$ be the Hardy space over the region $\mathscr{D}_{d}$, that is, the set of functions such that

$$
\lim _{\varepsilon \rightarrow 0} \int_{\partial \mathscr{D}_{d}(\varepsilon)}|u(z)||d z|<\infty .
$$

One then gets the following convergence results.

Theorem 3 (see [15]). Assume, with positive constants $\alpha, \beta$, and $d$, that

(1) $u$ belongs to $H^{1}\left(\mathscr{D}_{d}\right)$;

(2) $u$ decays exponentially on the real line; that is,

$$
|u(x)| \leq \alpha \exp (-\beta|x|), \quad \forall x \in \mathbb{R} .
$$

Then we have

$$
\begin{gathered}
\sup _{-\infty<x<\infty}\left|u^{(n)}(x)-\sum_{j=-N}^{N} u_{j} S_{j}^{(n)}(x)\right| \\
\leq C_{1} N^{(n+1) / 2} \exp (-\sqrt{\pi d \beta N}), \\
\sup _{-\infty<x<\infty}\left|\mathscr{H}[u](x)-\sum_{j=-N}^{N} u_{j} T_{j}(x)\right| \\
\leq C_{2} N^{1 / 2} \exp (-\sqrt{\pi d \beta N}),
\end{gathered}
$$

for some constants $C_{1}$ and $C_{2}$, where the mesh size $h$ is taken as

$$
h=\left(\frac{\pi d}{\beta N}\right)^{1 / 2} .
$$

The proof of the above Theorem 3 is beyond the scope of this paper. We refer the reader to [15] for a detailed discussion. Theorem 3 states that, if $u$ is an analytic function on an infinite strip containing the real line and allows specific growth restrictions, then it has exponentially decaying absolute errors in the Sinc approximation. Similarly, the approximation of the derivatives and the Hilbert transform of $u$ decay exponentially.

\section{Construction of the Method}

We consider the initial value problem for the Benjamin-Ono equation of the form

$$
u_{t}+u u_{x}+\mathscr{H}\left(u_{x x}\right)=0, \quad(x, t) \in \mathbb{R}^{2},
$$

together with the following initial:

$$
u(x, 0)=u_{0}(x)
$$

and the boundary conditions

$$
\begin{aligned}
& u-u_{x}=0 \quad \text { as } x \longrightarrow-\infty, \\
& u+u_{x}=0 \quad \text { as } x \longrightarrow \infty .
\end{aligned}
$$

Here $u: \mathbb{R}^{2} \rightarrow \mathbb{R}$ is a real valued function and $\mathscr{H}$ denotes the Hilbert transform defined by

$$
\mathscr{H}[f](x)=\frac{1}{\pi} P \int_{-\infty}^{\infty} \frac{f(x-y)}{y} d y,
$$

where $P$ denotes the Cauchy principal value.

In order to propose an unconditionally stable methodology, we discretize the time derivative of the $\mathrm{BO}$ equation using a classic finite difference formula by the $\theta$-weighted $(0 \leq \theta \leq 1)$ scheme between two successive levels $n$ and $n+1$ as

$$
\begin{aligned}
& \frac{u^{n+1}-u^{n}}{\delta t}+\theta\left(u u_{x}\right)^{n+1}+\theta \mathscr{H}\left(u_{x x}^{n+1}\right)+(1-\theta)\left(u u_{x}\right)^{n+1} \\
& +(1-\theta) \mathscr{H}\left(u_{x x}^{n+1}\right)=0
\end{aligned}
$$

where $u^{n}=u\left(x, t^{n}\right), t^{n}=t^{n-1}+\delta t$, and $\delta t$ is the time step size.

The nonlinear term $\left(u u_{x}\right)^{n+1}$ in (24) can be approximated by Taylor expansion. We obtain the following formula:

$$
\begin{aligned}
u^{n+1} & \approx u^{n}+\delta t\left(u_{t}\right)^{n} \\
& \approx u^{n}+\delta t\left(\frac{u^{n+1}-u^{n}}{\delta t}\right)+\mathcal{O}\left(\delta t^{2}\right), \\
\left(u_{x}\right)^{n+1} & \approx u_{x}^{n}+\delta t\left(\frac{u_{x}^{n+1}-u_{x}^{n}}{\delta t}\right)+\mathcal{O}\left(\delta t^{2}\right) .
\end{aligned}
$$

Therefore

$$
\begin{gathered}
\left(u u_{x}\right)^{n+1} \approx\left(u u_{x}\right)^{n}+\delta t\left[\left(u_{t}\right)^{n} u_{x}^{n}+u^{n} u_{x t}^{n}\right]+\mathcal{O}\left(\delta t^{2}\right) \\
=\left(u u_{x}\right)^{n}+\delta t\left[\left(\frac{u_{x}^{n+1}-u_{x}^{n}}{\delta t}\right) u_{x}^{n}\right. \\
\left.+u^{n}\left(\frac{u_{x}^{n+1}-u_{x}^{n}}{\delta t}\right)\right]+\mathcal{O}\left(\delta t^{2}\right), \\
=u^{n} u_{x}^{n+1}+u_{x}^{n} u^{n+1}-u^{n} u_{x}^{n}+\mathcal{O}\left(\delta t^{2}\right) .
\end{gathered}
$$


Equation (24) can be rewritten as

$$
\begin{aligned}
u^{n+1}+ & \delta t \theta\left[u^{n} u_{x}^{n+1}+u_{x}^{n} u^{n+1}+\mathscr{H}\left(u_{x x}^{n+1}\right)\right] \\
& =u_{n}-\delta\left[(1-2 \theta) u^{n} u_{x}^{n}+(1-\theta) \mathscr{H}\left(u_{x x}^{n}\right)\right] .
\end{aligned}
$$

Now the space variable is discretized into collocation points

$$
\begin{array}{r}
\left\{x_{1}=a, \ldots, x_{i}=a+(i-1) h, \ldots, x_{N}=b\right\}, \\
h=\frac{|b-a|}{N-1},
\end{array}
$$

so that the solution of (20) is interpolated and approximated by means of the Sinc functions as

$$
u\left(x, t^{n}\right)=u^{n}(x)=\sum_{j=1}^{N} u_{j}^{n} \xi_{j}(x),
$$

where the basis functions $\xi_{i}(x)$ are given by

$$
\xi_{j}(x)= \begin{cases}\omega_{0}(x), & j=1 \\ S_{j}(x), & j=2, \ldots, N-1, \\ \omega_{1}(x), & j=N .\end{cases}
$$

Here $S_{j}(x)$ is defined as

$$
S_{j}(x)=\operatorname{Sinc}\left(\frac{x-(j-1) h-a}{h}\right) \text {, }
$$

and the two cubic interpolants [14]

$$
\begin{aligned}
& \omega_{0}=\frac{(x-a)(b-x)^{2}}{(b-a)^{2}}+\frac{(2 x+b-3 a)(b-x)^{2}}{(b-a)^{3}}, \\
& \omega_{1}=\frac{(-2 x+3 b-a)(x-a)^{2}}{(b-a)^{3}}+\frac{(b-x)(x-a)^{2}}{(b-a)^{2}}
\end{aligned}
$$

are introduced to handle the radiation boundary conditions in (22).

The unknown parameters $u_{j}$ in (29) are to be determined by collocation method. Therefore, for each collocation point $x_{i}$, (29) can be written as

$$
u^{n}\left(x_{i}\right)=\sum_{j=1}^{N} u_{j}^{n} \xi_{j}\left(x_{i}\right), \quad i=1, \ldots, N
$$

The substitution of (33) into (27) at collocation points $x_{i}$ yields the following equation:

$$
\begin{aligned}
\sum_{j=1}^{N} u_{j}^{n+1} \xi_{j}\left(x_{i}\right)+\delta t \theta & {\left[\left(\sum_{j=1}^{N} u_{j}^{n} \xi_{j}\left(x_{i}\right)\right)\left(\sum_{j=1}^{N} u_{j}^{n+1} \xi_{j}^{\prime}\left(x_{i}\right)\right)\right.} \\
& +\left(\sum_{j=1}^{N} u_{j}^{n} \xi_{j}^{\prime}\left(x_{i}\right)\right)\left(\sum_{j=1}^{N} u_{j}^{n} \xi_{j}\left(x_{i}\right)\right) \\
& \left.+\sum_{i=1}^{N}\left(\sum_{j=1}^{N} u_{j}^{n+1} \xi^{\prime \prime}\left(x_{i}\right)\right) T_{i}\left(x_{k}\right)\right] \\
=\sum_{j=1}^{N} u_{j}^{n} \xi_{j}\left(x_{i}\right)-\delta t & {[1-2 \theta)\left(\sum_{j=1}^{N} u_{j}^{n} \xi_{j}\left(x_{i}\right)\right) } \\
& \times\left(\sum_{j=1}^{N} u_{j}^{n} \xi_{j}^{\prime}\left(x_{i}\right)\right) \\
& \left.+(1-\theta) \sum_{i=1}^{N}\left(\sum_{j=1}^{N} u_{j}^{n} \xi^{\prime \prime}\left(x_{i}\right)\right) T_{i}\left(x_{k}\right)\right] .
\end{aligned}
$$

To obtain the matrix representation of the expression (34), we introduce the following matrix and vector notations:

$$
\begin{aligned}
& u^{n}=\left[u_{1}^{n}, u_{2}^{n}, \ldots, u_{N}^{n}\right]^{T}, \\
& \mathbf{I}^{(0)}=\left(\xi_{j}\left(x_{i}\right)\right), \quad i, j=1, \ldots, N, \\
& \mathbf{I}^{(1)}=\left(\xi_{j}^{\prime}\left(x_{i}\right)\right), \quad i, j=1, \ldots, N, \\
& \mathbf{I}^{(2)}=\left(\xi_{j}^{\prime \prime}\left(x_{i}\right)\right), \quad i, j=1, \ldots, N, \\
& \mathbf{H}=\left(T_{j}\left(x_{i}\right)\right), \quad i, j=1, \ldots, N .
\end{aligned}
$$

Thus we obtain the following system of $N$ linear equations in $N$ unknown parameters $u^{n+1}$ which can be expressed in a matrix form

$$
\mathbf{M} u^{n+1}=\mathbf{R} u^{n}
$$

where

$$
\begin{aligned}
& \mathbf{M}=\mathbf{I}^{(0)}+\delta t \theta\{\mathbf{A}+\mathbf{B}+\mathbf{C}\} \\
& \mathbf{R}=\mathbf{I}^{(0)}-\delta t\{(1-2 \theta) \mathbf{D}+(1-\theta) \mathbf{C}\}
\end{aligned}
$$

in which

$$
\begin{array}{ll}
u_{x}^{n}=\mathbf{I}^{(1)} u^{n}, & \mathbf{A}=u^{n} * \mathbf{I}^{(1)}, \quad \mathbf{B}=u_{x}^{n} * \mathbf{I}^{(0)}, \\
\mathbf{C}=\mathbf{H} \times \mathbf{I}^{(2)}, & \mathbf{D}=u^{n} * u_{x}^{n} * \mathbf{I}^{(0)},
\end{array}
$$

where symbols "*" and " $x$ " mean the component-wise and the matrix multiplications, respectively. 
The stability of the approximation (36) is investigated by using the matrix method. The linearized version of (20) is obtained by assuming the quantity $u$ in the nonlinear term $u u_{x}$ as a local constant $\bar{u}$. The error at timestep $n$ is defined as

$$
e^{n}=u_{\text {exact }}^{n}-u_{\text {num }}^{n},
$$

where $u_{\text {exact }}^{n}$ and $u_{\text {num }}^{n}$ are the exact and the numerical solutions, respectively. The error matrix equation of the linearized $\mathrm{BO}$ equation is given by

$$
[\mathbf{I}+\theta \delta t \mathbf{J}] e^{n+1}=[\mathbf{I}+(1-\theta) \delta t \mathbf{J}] e^{n},
$$

where $\mathbf{I}=\mathbf{I}^{(0)}$ and $\mathbf{J}=\bar{u} \mathbf{I}^{(1)}+\mathbf{H} \times \mathbf{I}^{(2)}$. Now, let $\mathbf{P}=[\mathbf{I}+$ $\theta \delta t \mathbf{J}]^{-1}[\mathbf{I}+(1-\theta) \delta t \mathbf{J}]$; then (40) becomes

$$
e^{n+1}=\mathbf{P} e^{n}
$$

The numerical scheme is stable if $\|\mathbf{P}\|_{2} \leqslant 1$, or equivalently, $\rho(\mathbf{P}) \leqslant 1$; that is, the spectral radius of the matrix $\mathbf{P}$ has to be smaller than or equal to one. From (40) it can be seen that the stability conditions are possible if all the eigenvalues of the matrix $[\mathbf{I}+\theta \delta t \mathbf{J}]^{-1}[\mathbf{I}+(1-\theta) \delta t \mathbf{J}]$ satisfy the following condition:

$$
\left|\frac{\lambda_{I}-\delta t(1-\theta) \lambda_{J}}{\lambda_{I}+\delta t \theta \lambda_{J}}\right| \leqslant 1,
$$

where $\lambda_{I}$ and $\lambda_{J}$ are the eigenvalues of the matrices $I$ and $J$, respectively.

For $\theta=0.5$, the relation (42) becomes

$$
\left|\frac{\lambda_{I}-0.5 \delta t \lambda_{J}}{\lambda_{I}+0.5 \delta t \lambda_{J}}\right| \leqslant 1
$$

If both $\lambda_{I}$ and $\lambda_{J}$ are complex eigenvalues, we write $\lambda_{I}=a_{h}+$ $i b_{h}$ and $\lambda_{J}=a_{k}+i b_{k}$. In this case, (43) can be rewritten as

$$
\left|\frac{\left(a_{h}-0.5 \delta t a_{k}\right)+i\left(b_{h}-0.5 \delta t b_{k}\right)}{\left(a_{h}+0.5 \delta t a_{k}\right)+i\left(b_{h}+0.5 \delta t b_{k}\right)}\right| \leqslant 1 .
$$

The inequality (44) is satisfied if $a_{h} a_{k}+b_{h} b_{k} \geqslant 0$. For real eigenvalues, the inequality (44) holds true for $\left(\lambda_{h} \geqslant 0\right.$ and $\left.\lambda_{h} \geqslant 0\right)$ or $\left(\lambda_{h} \leqslant 0\right.$ and $\left.\lambda_{h} \leqslant 0\right)$. This shows that scheme (36) is unconditionally stable if $a_{h} a_{k}+b_{h} b_{k} \geqslant 0$ for complex eigenvalues and $\left(\lambda_{h} \geqslant 0\right.$ and $\left.\lambda_{h} \geqslant 0\right)$ or $\left(\lambda_{h} \leqslant 0\right.$ and $\left.\lambda_{h} \leqslant 0\right)$ for real eigenvalues.

For $\theta=0$, the inequality (42) becomes

$$
\left|1-\frac{\delta t \lambda_{J}}{\lambda_{I}}\right| \leqslant 1
$$

In other words,

$$
\delta t \leqslant \frac{2 \lambda_{J}}{\lambda_{I}}, \quad \frac{\lambda_{J}}{\lambda_{I}} \geqslant 0 .
$$

Therefore, when $\theta=0$, the scheme (42) is conditionally stable.

\section{Numerical Results}

The analytical soliton solutions of the $\mathrm{BO}$ equation are rational functions in $x$ and $t$. For example, one and two soliton solutions are given, respectively, by $($ see $[6,16,17])$

$$
\begin{gathered}
u(x, t)=\frac{4 k_{1}}{k_{1}^{2} \theta_{1}^{2}+1}, \\
u(x, t) \\
=\frac{4 k_{1} k_{2}\left(k_{1} \theta_{1}^{2}+k_{2} \theta_{2}^{2}+k_{1}^{-1} k_{2}^{-1}\left(k_{1}+k_{2}\right)^{3}\left(k_{1}-k_{2}\right)^{-2}\right)}{\left(k_{1} k_{2}-\left(k_{1}+k_{2}\right)^{2}\left(k_{1}-k_{2}\right)^{-2}\right)^{2}+\left(k_{1} \theta_{1}+k_{2} \theta_{2}\right)^{2}}
\end{gathered}
$$

where $\theta_{1}=x-k_{1} t+\phi_{1}, \theta_{2}=x-k_{2} t+\phi_{2}$, and $k_{1}, k_{2}, \phi_{1}$, $\phi_{2}$ are arbitrary constants. In this section, we examine the proposed algorithm using different test problems related to the propagation of one soliton and interaction of two soliton solutions. The accuracy of the scheme is measured by using the following error norms:

$$
\begin{gathered}
\|E\|_{2}=\|u-\tilde{u}\|_{2}=\left[h \sum_{j=1}^{N}\left(u_{j}-\tilde{u}_{j}\right)^{2}\right]^{1 / 2}, \\
\|L\|_{\infty}=\|u-\tilde{u}\|_{\infty}=\max _{1 \leqslant j \leqslant N}\left|u_{j}-\tilde{u}_{j}\right|,
\end{gathered}
$$

where $u$ and $\tilde{u}$ represent the exact and approximate solutions, respectively, and $h$ is the minimum distance between any two points of set points for which the errors are evaluated. In our computational work, we use the collocation points

$$
\left\{x_{1}=a, \ldots, x_{i}=a+(i-1) h, \ldots, x_{N}=b\right\}, \quad h=\frac{|b-a|}{N-1} .
$$

The $\mathrm{BO}$ equation possesses infinite conservation laws [5]; the first three are given as follows:

$$
\begin{aligned}
& I_{1}=\int_{a}^{b} u d x, \quad I_{2}=\int_{a}^{b} u^{2} d x, \\
& I_{3}=\int_{a}^{b}\left[u^{3}-3 u_{x} \mathscr{H}(u)\right] d x,
\end{aligned}
$$

related to the mass, momentum, and energy. The quantities $I_{1}$, $I_{2}$, and $I_{3}$ are applied to measure the conservation properties of the collocation scheme calculated by

$$
\begin{aligned}
& I_{1} \approx h \sum_{j=1}^{N} u_{j}^{n}, \quad I_{2} \approx h \sum_{j=1}^{N}\left(u_{j}^{n}\right)^{2}, \\
& I_{3} \approx h \sum_{j=1}^{N}\left[\left(u_{j}^{n}\right)^{3}-3\left(u_{x}\right)_{j}^{n} \mathscr{H}\left(u_{j}^{n}\right)\right] .
\end{aligned}
$$




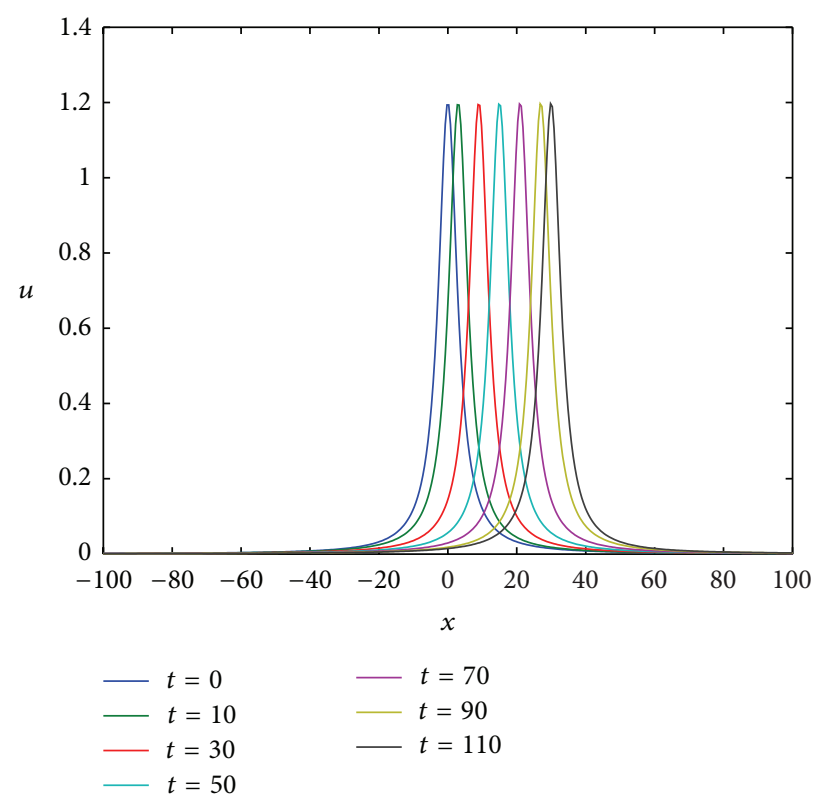

(a)

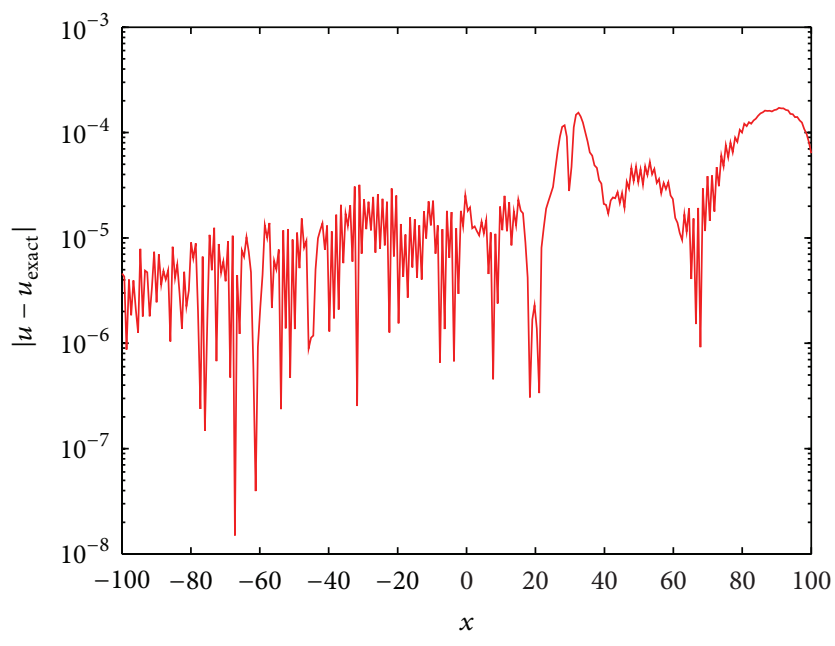

FIGURE 1: Propagation of soliton solutions (a) and absolute error (b) at $t=110$ of the BO equation $k_{1}=0.3, \phi_{1}=0, N=200, \delta t=0.001$, $\theta=1 / 2$, and $x \in[-100,100]$.

TABLE 1: Invariants and errors for a single soliton of the BO equation. $k_{1}=0.3, \phi_{1}=0, N=200, \delta t=0.001, \theta=1 / 2$, and $x \in[-100,100]$.

\begin{tabular}{lcccccc}
\hline Time & $L_{\infty}$ & $L_{2}$ & $I_{1}$ & $I_{2}$ & $I_{3}$ & CPU(s) \\
\hline 0 & 0 & 0 & 12.3007 & 7.5397 & 0.000 \\
10 & $3.3754 E-5$ & $7.6966 E-5$ & 12.3007 & 7.5397 & 1788 \\
30 & $6.0085 E-5$ & $1.8419 E-4$ & 12.3008 & 7.5397 & 10.1788 \\
50 & $8.5661 E-5$ & $3.0307 E-4$ & 12.3008 & 7.5397 & 10.1788 \\
70 & $1.1471 E-4$ & $4.5713 E-4$ & 12.3008 & 7.5397 & 10.1788 \\
90 & $1.5029 E-4$ & $6.5433 E-4$ & 12.3008 & 7.5397 & 10.1788 \\
110 & $1.9803 E-4$ & $9.3644 E-4$ & 12.3008 & 7.5397 & 10.1788 & 3.605 \\
\hline
\end{tabular}

4.1. Propagation of Single Solitons. In this experiment, we consider the propagation of single solitons of the BO equation. The initial and boundary conditions are extracted from (47) and (22), respectively. The values of the parameters used in the numerical experiments are $k_{1}=0.3, \phi_{1}=0, \theta=1 / 2$, the number of collocation points $N=200$, and $\delta t=0.001$. The soliton from which the initial condition is extracted (47) moves to the right across the space interval $x \in[-100,100]$ when the time interval is $t \in[0,110]$. The numerical tests for this case are performed using the Sinc basis functions.

The error norms $L_{\infty}, L_{2}$ and conservation quantities $I_{1}$, $I_{2}$, and $I_{3}$ are computed, which are shown in Table 1. From the numerical results given in Table 1 it is observed that, throughout the simulation, the error norms $L_{\infty}$ remain less than $1.9803 \times 10^{-4}$ and $L_{2}$ remains less than $9.3644 \times 10^{-4}$, whereas the changes of the invariant $I_{1}$ are less than $10^{-4}$ and the invariants $I_{2}$ and $I_{3}$ at a given time $t$ are equal to those of the initial value; our scheme is satisfactorily conservative. The numerical solutions at different time levels (a) and the absolute error (b) at time $t=110$ are shown in Figure 1 for $k_{1}=0.3, \phi_{1}=0, \theta=1 / 2$, the number of collocation points $N=200$, and $\delta t=0.001$ in the region $x \in[-100,100]$.

In the next experiment, we investigate the convergence of our approach in terms of the number of grid points $N$ at time $t=1$. To ensure that our scheme is not dominated by the time discretization, we choose $\delta t=10^{-5}$. We take the parameters such as $k_{1}=0.4, k_{2}=0.1, \phi_{1}=30, \phi_{2}=0, \delta t=10^{-5}, \theta=1 / 2$, and $x \in[-100,100]$.

Figure 2 shows that the error norms $L_{\infty}$ and $L_{2}$ converge exponentially for the propagation of one soliton solutions and for the interaction of two soliton solutions as we increase the number of grid points $N$.

4.2. Interaction of Two Solitons. Our second experiment pertains to the interaction of two soliton solutions of the BO equation having different amplitudes and travelling in the same direction. The initial and boundary conditions are extracted from (47) and (22).

To allow the interaction to occur, the experiment was run from $t=0$ to 210 in the region $[-100,100]$. Figure 3 


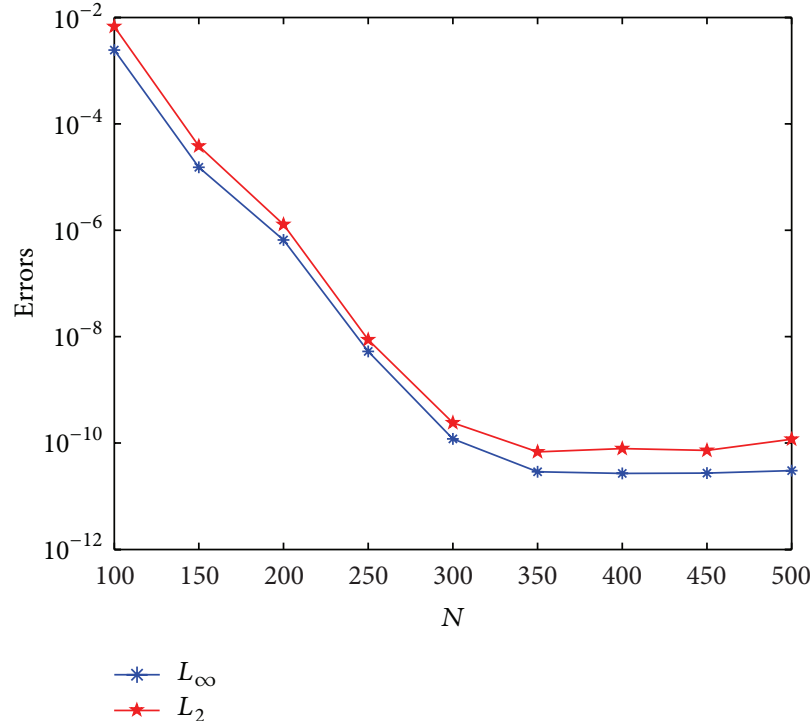

(a)

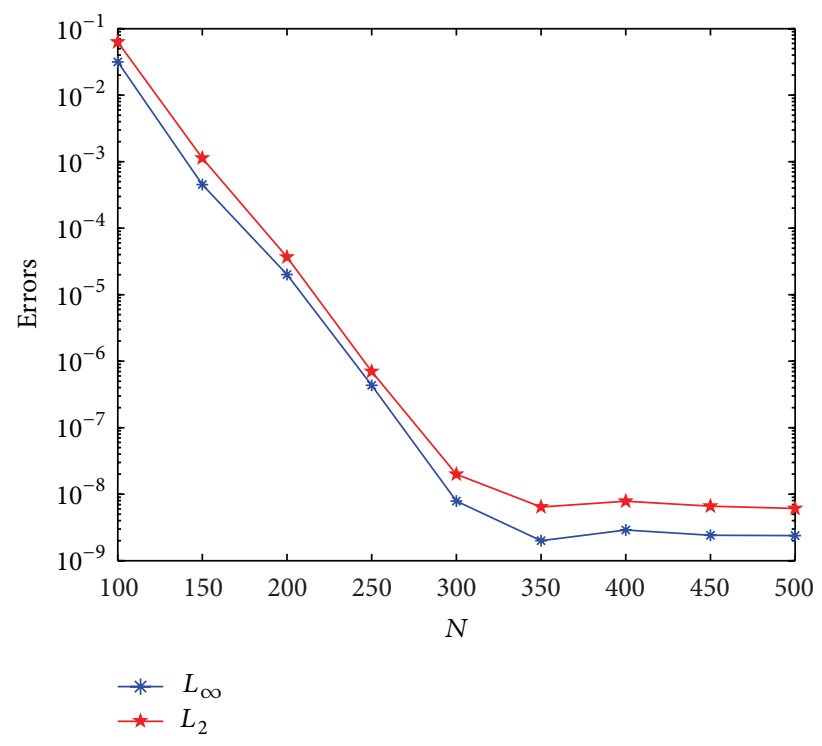

(b)

FIGURE 2: Errors for the propagation of one soliton solutions (a) and for the interaction of two soliton solutions (b) at $t=1$ of the BO equation with $k_{1}=0.4, k_{2}=0.1, \phi_{1}=30, \phi_{2}=0, \delta t=10^{-5}, \theta=1 / 2$, and $x \in[-100,100]$.

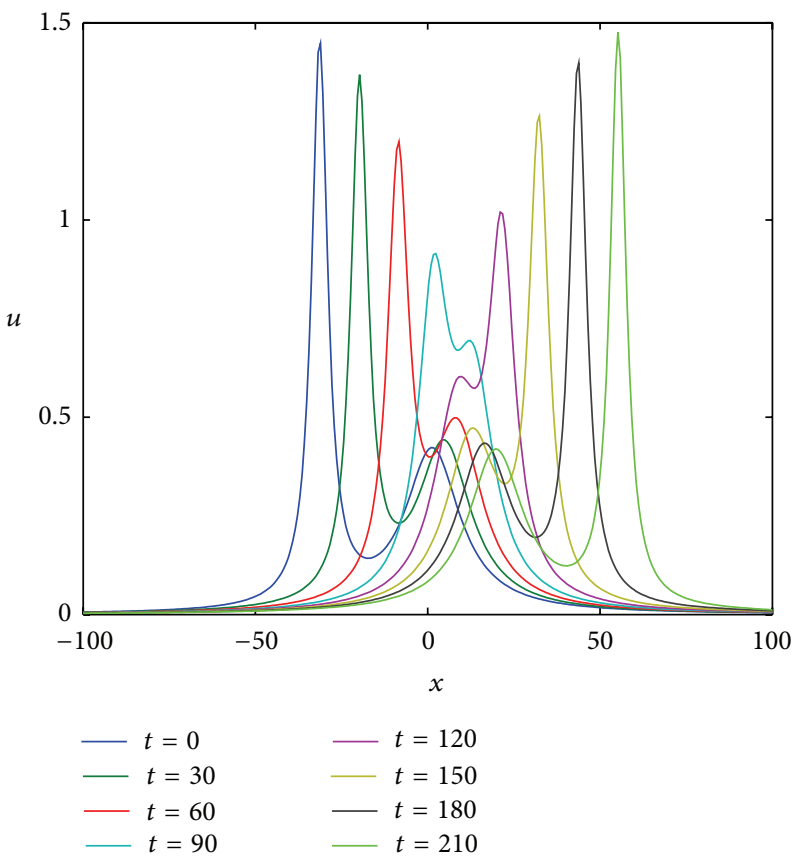

(a)

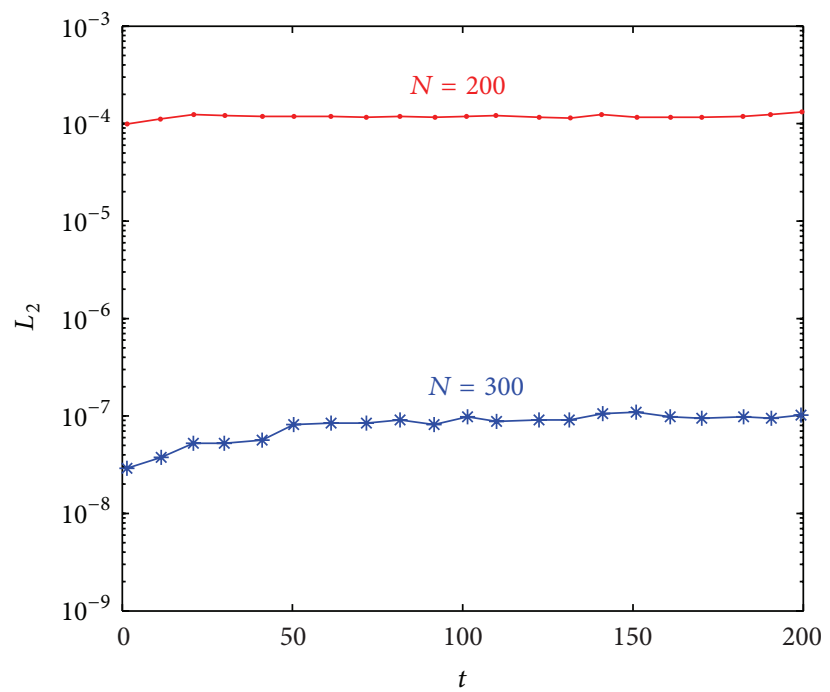

(b)

Figure 3: Time evolution of the interaction of two soliton solutions (a) and $L_{2}$ error (b) of the BO equation. $k_{1}=0.4, k_{2}=0.1, \phi_{1}=30$, $\phi_{2}=0, N=200$ and $300, \delta t=0.001, \theta=1 / 2$, and $x \in[-100,100]$.

shows the interaction of two soliton solutions of $\mathrm{BO}$ equation for $k_{1}=0.4, k_{2}=0.1, \phi_{1}=30, \phi_{2}=0, N=200$, $\delta t=0.001, \theta=1 / 2$, and $x \in[-100,100]$. It can be seen that the faster pulse interacts with and emerges ahead of the lower pulse with the shape and velocity of each soliton retained. Because of the nature of the Sinc discretization, the Hilbert transform and matrix differentiation are explicitly obtained with exponential accuracy. The result in Figure 3 shows numerical interaction of the two soliton solution and its time evolution of the $L_{2}$ error from $t=0$ to $t=$ 210. In Figure 3(b), it is shown that the Sinc discretization maintains accurate results comparable to those given in [7, 10] for a long period. Therefore, a number of properties of the Benjamin-Ono equation can be checked numerically, 
TABLE 2: Invariants and errors for interaction of two solitons of the BO equation. $k_{1}=0.4, k_{2}=0.1, \phi_{1}=30, \phi_{2}=0, N=200, \delta t=0.001$, $\theta=1 / 2$, and $x \in[-100,100]$.

\begin{tabular}{lccccc}
\hline Time & $L_{\infty}$ & $L_{2}$ & $I_{1}$ & $I_{2}$ & $I_{3}$ \\
\hline 0 & 0 & 0 & 24.0123 & 12.5716 & 16.7526 \\
1 & $1.0200 E-4$ & $1.4299 E-4$ & 24.0124 & 12.5716 & 16.7526 \\
30 & $2.7172 E-4$ & $8.2385 E-4$ & 24.0124 & 12.5716 & 16.7526 \\
60 & $4.0996 E-4$ & $1.4813 E-3$ & 24.0124 & 12.5716 & 16.7526 \\
90 & $5.6586 E-4$ & $2.1770 E-3$ & 24.0124 & 12.5716 & 16.7526 \\
120 & $6.8573 E-4$ & $2.9978 E-3$ & 24.0124 & 12.5716 & 16.7526 \\
150 & $8.5960 E-4$ & $4.2343 E-3$ & 24.0124 & 12.5716 & 16.833 \\
180 & $2.1269 E-3$ & $6.6914 E-3$ & 24.0124 & 12.5716 & 1.8526 \\
210 & $3.1148 E-3$ & $9.6304 E-3$ & 24.0124 & 12.5716 & 16.7526 \\
\hline
\end{tabular}

including the exhibition of no phase shifts after interaction of the solitons.

Numerical check on the conservation mass, momentum, and energy shows that the three quantities remain constant with respect to time as shown in Table 2. Propagation of the single solitary wave and two soliton integrations are simulated well with the proposed algorithms and conservation invariants do not change much during the computer run. Thus Sinc functions can be used to construct approximate numerical methods of the $\mathrm{BO}$ equation.

\section{Conclusion}

A numerical technique based on the Sinc collocation method has been presented for numerical solutions of the BO equation. The efficiency of the method is tested on the problems of propagation of a single soliton as well as interaction of two solitons. The accuracy of solutions is examined in terms of the $L_{\infty}, L_{2}$ error norms, and the conservation quantities $I_{1}, I_{2}$, and $I_{3}$. Stability analysis is performed by the matrix method. The results obtained for the Sinc collocation are very close to analytical ones. Our algorithm was found to be unconditionally stable and exponentially convergent in space and a reliable numerical method for solving the $\mathrm{BO}$ equation. The method is easy to implement and compares favourably to Fourier-based spectral methods without any assumptions on periodicity.

\section{Conflict of Interests}

The authors declare that there is no conflict of interests regarding the publication of this paper.

\section{Acknowledgment}

Edson Pindza is thankful to Brad Welch for the financial support from RidgeCape Capital.

\section{References}

[1] J. A. C. Weideman, "Computing the Hilbert transform on the real line," Mathematics of Computation, vol. 64, no. 210, pp. 745762, 1995.
[2] T. B. Benjamin, "Internal waves of permanent form in fluids of great depth," Journal of Fluid Mechanics, vol. 29, no. 3, pp. 559$592,1967$.

[3] H. Ono, "Algebraic solitary waves in stratified fluids," Journal of the Physical Society of Japan, vol. 39, no. 4, pp. 1082-1091, 1975.

[4] C. E. Kenig, G. Ponce, and L. Vega, "Well-posedness and scattering results for the generalized Korteweg-de Vries equation via the contraction principle," Communications on Pure and Applied Mathematics, vol. 46, no. 4, pp. 527-620, 1993.

[5] L. Abdelouhab, J. L. Bona, M. Felland, and J. Saut, "Nonlocal models for nonlinear, dispersive waves," Physica D, vol. 40, no. 3, pp. 360-392, 1989.

[6] J. Satsuma and Y. Ishimori, "Periodic wave and rational soliton solutions of the Benjamin-Ono equation," Journal of the Physical Society of Japan, vol. 46, no. 2, pp. 681-687, 1979.

[7] R. L. James and J. A. C. Weideman, "Pseudospectral methods for the Bejamin-Ono equation," in Advances in Computer Methods for Partial Differential Equations VII, pp. 371-377, 1992.

[8] T. Miloh, M. Prestin, L. Shtilman, and M. P. Tulin, "A note on the numerical and $N$-soliton solutions of the Benjamin-Ono evolution equation," Wave Motion, vol. 17, no. 1, pp. 1-10, 1993.

[9] V. Thomée and A. S. Vasudeva Murthy, "A numerical method for the Benjamin-Ono equation," BIT Numerical Mathematics, vol. 38, no. 3, pp. 597-611, 1998.

[10] J. P. Boyd and Z. Xu, "Comparison of three spectral methods for the Benjamin-Ono equation: Fourier pseudospectral, rational Christov functions and Gaussian radial basis functions," Wave Motion, vol. 48, no. 8, pp. 702-706, 2011.

[11] F. Stenger, "A Sinc-Galerkin methods for solution of boundary value problems," Mathematics of Computation, vol. 33, pp. 85109, 1979.

[12] K. Al-Khaled, "Sinc numerical solution for solitons and solitary waves," Journal of Computational and Applied Mathematics, vol. 130, no. 1-2, pp. 283-292, 2001.

[13] R. Mokhtari and M. Mohammadi, "Numerical solution of GRLW equation using Sinc-collocation method," Computer Physics Communications, vol. 181, no. 7, pp. 1266-1274, 2010.

[14] J. Lund and K. L. Bowers, Sinc Methods For Quadrature and Differential Equations, SIAM, Philadelphia, Pa, USA, 1992.

[15] F. Stenger, Numerical Methods Based on Sinc and Analytic Functions, Springer, New York, NY, USA, 1993.

[16] Y. Matsuno, "Exact multi-soliton solution of the Benjamin-Ono equation," Journal of Physics A: Mathematical and General, vol. 12, no. 4, pp. 619-621, 1979.

[17] Y. Matsuno, "Interaction of the Benjamin-Ono solitons," Journal of Physics A: Mathematical and General, vol. 13, no. 5, pp. 1519$1536,1980$. 

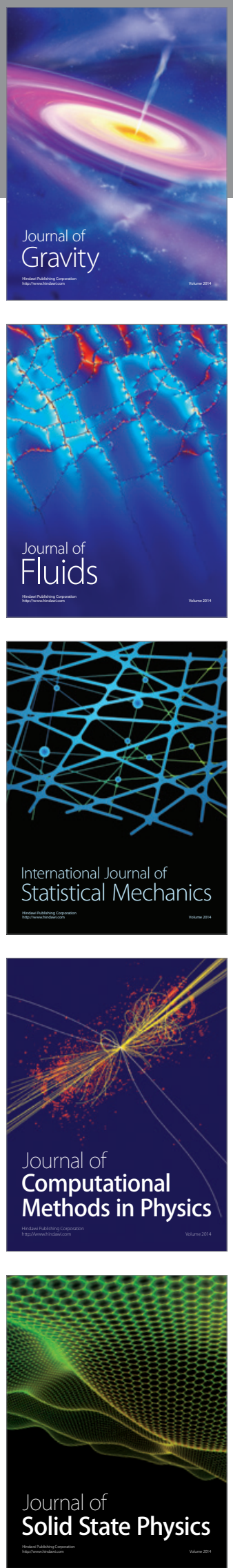

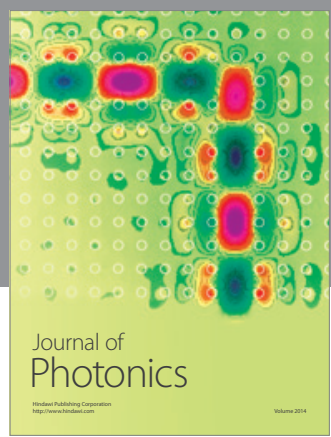

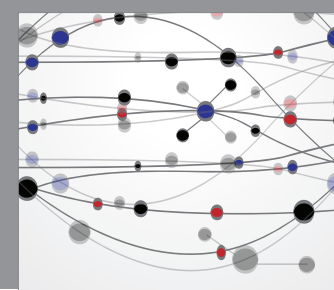

The Scientific World Journal

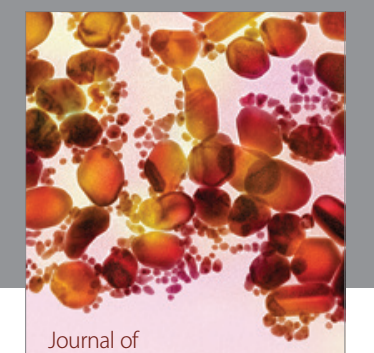

Soft Matter
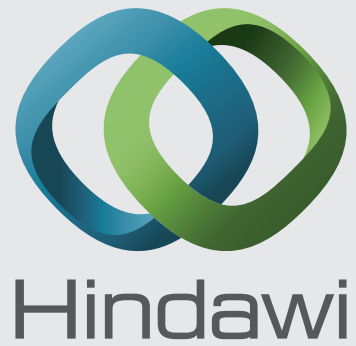

Submit your manuscripts at

http://www.hindawi.com
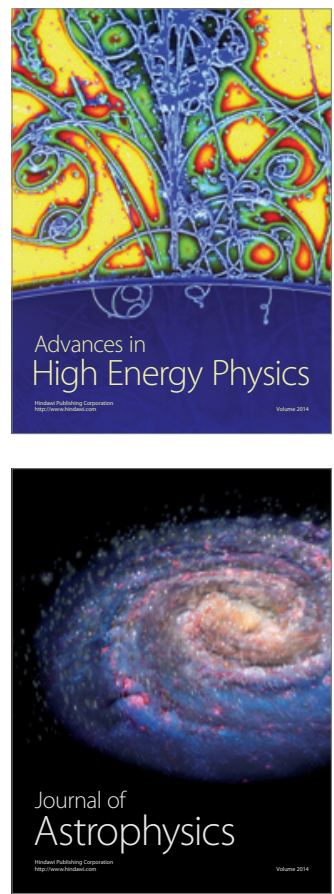
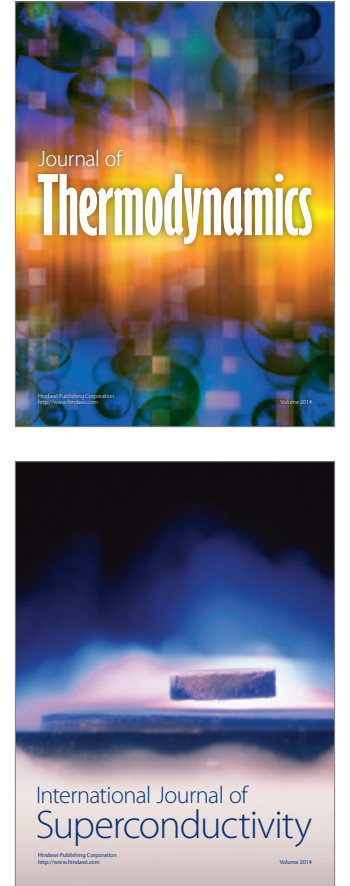
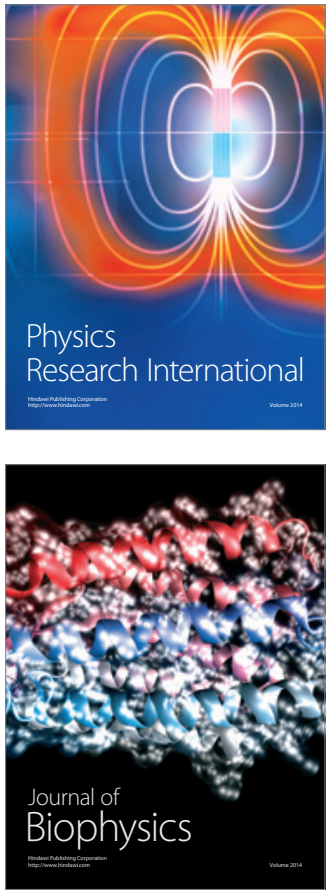
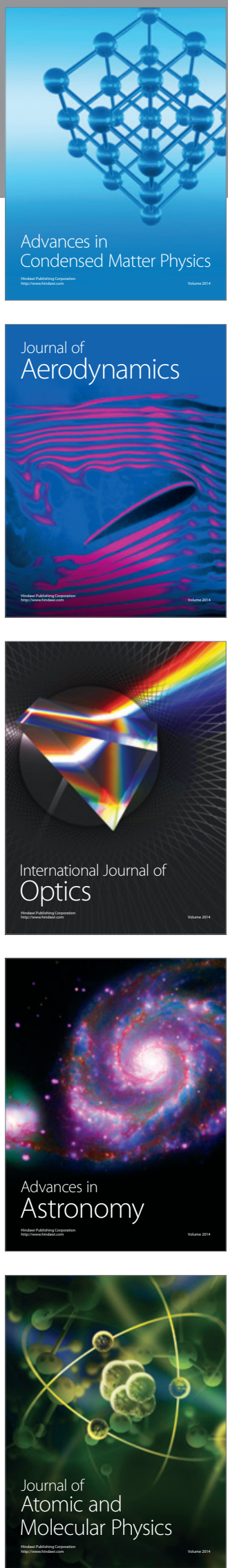